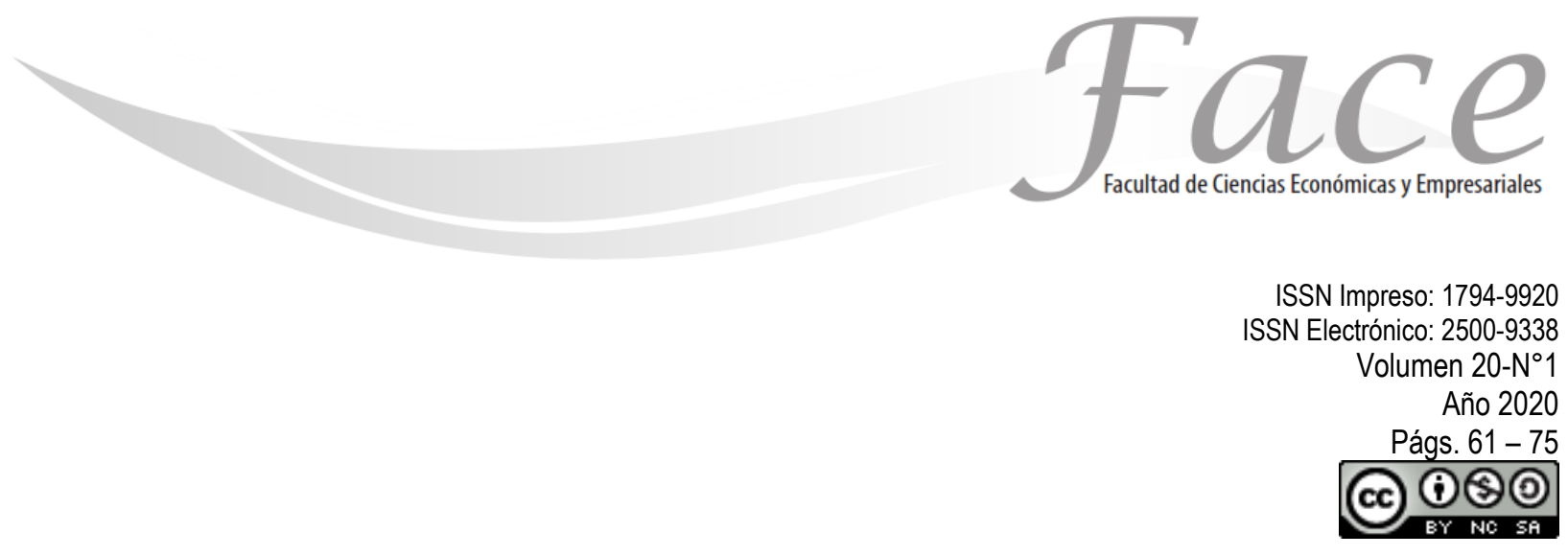

\title{
INDICADORES DE RENTABILIDAD, ENDEUDAMIENTO Y EBITDA EN EL ENTORNO DE LA INVERSIÓN EN LAS PLATAFORMAS TECNOLÓGICAS. UN ESTUDIO EN ADMINISTRADORAS DE FONDOS DE PENSIONES
}

\author{
Campo Elías López-Rodríguez * \\ https://orcid.org/0000-0003-4061-2979 \\ Lina Marcela Pérez-Suarez ** \\ https://orcid.org/0000-0003-0908-2965 \\ Francisco Javier Amado-Quiroga *** \\ https://orcid.org/0000-0002-6875-5560 \\ Yeison Castillo-Areiza ${ }^{* * *}$ \\ https://orcid.org/0000-0002-4650-906X
}

Fecha de Recepción: Abril 2 de 2020

Fecha de Aprobación: Julio 1 de 2020

\section{Resumen:}

El propósito del artículo es analizar los indicadores de rentabilidad ROA y ROE, endeudamiento y EBITDA generados por la inversión de plataformas tecnológicas en una Administradora de Fondos de Pensiones. Desde un alcance descriptivo, enfoqué cualitativo y a través de estudio de caso se pudo determinar la importancia de realizar este tipo de inversiones analizando cómo influyen los indicadores en la generación de valor de los activos. Los resultados determinan que al realizar la inversión hubo un aumento significativo para los dos últimos años estudiados teniendo en cuenta tanto los ingresos, costos y gastos generados durante este periodo de tiempo.

Palabras clave: Indicadores financieros, inversión, plataformas tecnológicas, Administradoras de Fondos de Pensiones.

\footnotetext{
* Magister en Gerencia Estratégica de Mercadeo. Líder de Investigación del programa de Especialización en Gerencia Financiera de la Corporación Universitaria Minuto de Dios - UNIMINUTO - Colombia Contacto: clopezr3@uniminuto.edu.co

** Magister en Finanzas. Docente investigadora del programa Administración Financiera de la Corporación Universitaria Minuto de Dios - UNIMINUTO -Colombia. Contacto: lina.perezs@uniminuto.edu

*** Especialista en Gerencia Financiera de la Corporación Universitaria Minuto de Dios - UNIMINUTO - Colombia. Contacto: jeravergara67@gmail.com

${ }^{* * * \star}$ Especialista en Gerencia Financiera de la Corporación Universitaria Minuto de Dios - UNIMINUTO- Colombia. Contacto: yeison.castillo@uniminuto.edu.co
} 


\title{
INDICATORS OF PROFITABILITY, DEBT AND EBITDA IN THE ENVIRONMENT OF INVESTMENT IN TECHNOLOGICAL PLATFORMS. A STUDY IN PENSION FUND MANAGERS
}

\begin{abstract}
:
The purpose of the article is to analyze ROA and ROE profitability indicators, indebtedness and EBITDA generated by the investment of technological platforms in a Pension Fund Administrator. From a descriptive scope, I focused qualitatively and through a case study it was possible to determine the importance of making this type of investment by analyzing how the indicators influence the generation of asset value. The results determine that when making the investment there was a significant increase for the last two years studied, taking into account both the income, costs and expenses generated during this period of time.
\end{abstract}

Keywords: Financial indicators, investment, technological platforms, Pension Fund Administrators.

\section{INDICADORES DE RENTABILIDADE, DÍVIDA E EBITDA NO AMBIENTE DE INVESTIMENTO EM PLATAFORMAS TECNOLÓGICAS. UM ESTUDO EM GESTORES DE FUNDOS DE PENSÃO}

\author{
Resumo:
}

O objetivo do artigo é analisar os indicadores de rentabilidade, ROA e ROE, endividamento e EBITDA gerados pelo investimento de plataformas tecnológicas em um Administrador de Fundos de Pensão. Do ponto de vista descritivo, concentrei-me qualitativamente e, através de um estudo de caso, foi possível determinar a importância de realizar esse tipo de investimento, analisando como os indicadores influenciam a geração de valor do ativo. Os resultados determinam que, ao realizar o investimento, houve um aumento significativo nos últimos dois anos estudados, levando em consideração a receita, os custos e as despesas geradas durante esse período.

Palavras-chave: Indicadores financeiros, investimentos, plataformas tecnológicas, Administradores de fundos de pensão. 


\section{INTRODUCCIÓN:}

Las administradoras de fondos de pensiones (AFP) son instituciones privadas financieras creadas con la Ley 100 de 1993 con el objetivo de ofrecer una gestión eficiente administrando los fondos y planes de pensiones de ahorro individual con solidaridad y de los fondos de cesantías en Colombia; dichas compañías para mantenerse y crecer en el sector deben realizar inversiones en procesos tecnológicos ofreciendo a sus clientes un mayor dinamismo en el tiempo de respuesta y servicio al cliente (López-Rodríguez, Cristancho-Triana \& Amaya-Téllez, 2020).

Teniendo en cuenta que las organizaciones necesitan parámetros de evaluación para reconocer el estado real de sus rendimientos desde las inversiones (LópezRodríguez et al, 2019), es relevante tener un acercamiento hacia la manera en la cual, desde una perspectiva financiera, estos activos pueden ser controlados y manejados por las directivas de las AFP. Ante ello, los indicadores de rentabilidad son un mecanismo financiero que tienen como función el evidenciar el nivel de eficiencia con el cual se manejan los activos promedio dentro de las organizaciones (Andrade-Pinelo, 2011).

A partir del uso adecuado dentro de la gerencia financiera de este indicador las empresas podrán encontrar la rentabilidad de sus activos, en especial los soportados en la tecnología. El ROA al ser una ratio que va más allá de los beneficios o cifras de facturación, es útil para monitorear el desarrollo financiero de las organizaciones ayudando a predecir una estrategia interna en futuras inversiones. De igual, manera es necesario evaluar el EBITDA de una empresa, ya que si este indicador es positivo revela que el proyecto tiene alta probabilidad de ser exitoso y el resultado dependerá de los gastos financieros ejecutados.
Un indicador que es necesario tener en cuenta en las razones financieras es el análisis del endeudamiento, con él se evidencia el nivel de apalancamiento a corto y largo plazo y la capacidad de endeudamiento que tiene una organización. Es por esto por lo que al estudiar adecuadamente el endeudamiento contable se determina cuándo y cómo se puede apalancar en un momento determinado cualquier organización, apreciado únicamente desde el punto de vista financiero (García, 2018).

Es relevante para la gerencia financiera contar con alternativas para su control, como es el caso del ROA, que se caracteriza por identificar el comportamiento de los activos intangibles y el retorno que pueda llegar a generar la compañía (Pérez-Rave, Ocampo \& Restrepo, 2018). De igual manera, Andrade-Pinelo (2011) menciona que este indicador ha sido utilizado en las organizaciones con el propósito de analizar las variables y buscar un manejo eficiente de la rentabilidad y medir de manera comparativa los resultados obtenidos contra distintos niveles de inversión, sobre las cuales se busca tener control financiero en la empresa.

Por lo anterior, es pertinente analizar desde el ROA, ROE, endeudamiento y EBITDA los resultados entregados por la inversión realizada en innovación de canales electrónicos en servicio al cliente por parte de la AFP desde la cual se desarrollará el presente estudio, y así conocer impactos positivos o beneficios de dicha inversión. En la figura 1 se evidencia el número de afiliados en el año 2017 dividido según el fondo de pensiones y cesantías y la segmentación por el fondo interno de inversión. De ella se analiza que hay una elección mayoritaria por Porvenir versus la competencia nombrada, pues abarca más del $50 \%$ del mercado. 
Figura 1. Número de afiliados en AFP al año 2017

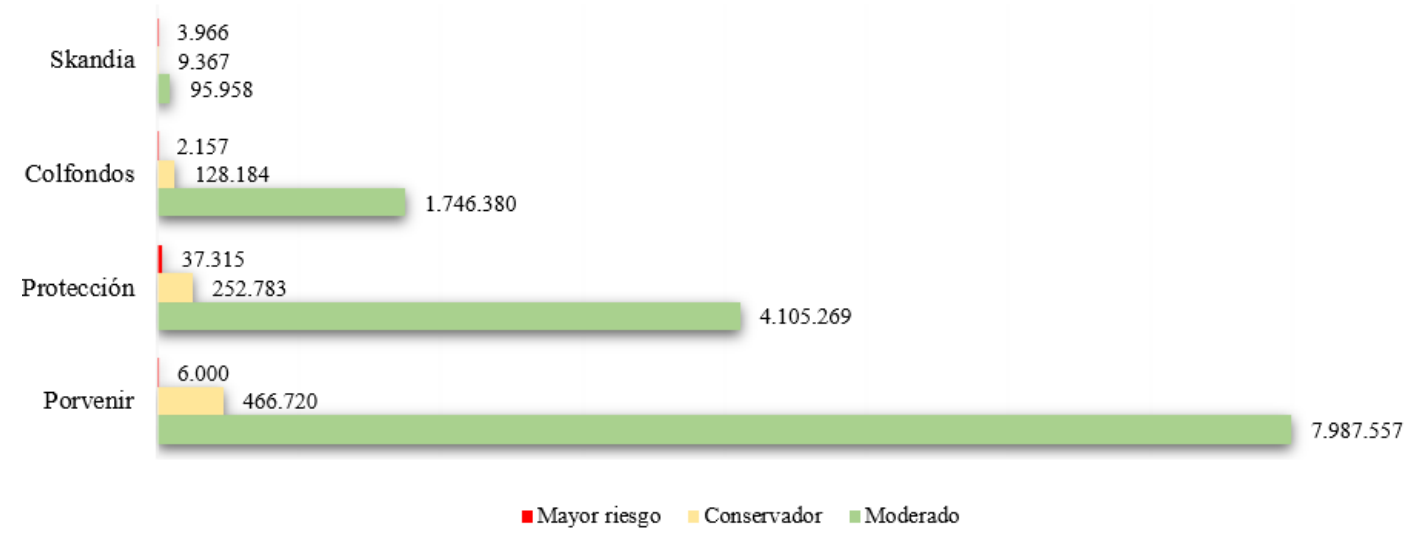

Fuente: Elaboración propia con base en la Superintendencia Financiera (2018)

La administradora de fondo de pensiones y cesantías estudiada, con los resultados entregados podrá concluir si los recursos financieros invertidos generaron rentabilidad a futuro, también se concluirá si para ella la inversión en la innovación generó valor agregado, teniendo en cuenta que en los últimos años la innovación tecnológica genera posicionamiento en el mercado por el ahorro de tiempo de respuesta.

Teniendo en cuenta lo anterior, el objetivo general de la presente investigación es el de analizar los indicadores de rentabilidad ROA y ROE, endeudamiento y EBITDA en la generación de valor de los activos, a partir de la inversión en las plataformas tecnológicas en una organización del sector financiero colombiano. Para poder dar alcance a lo anterior, se hace necesario relacionar los conceptos de los indicadores ROA y ROE, endeudamiento y EBITDA para el análisis de la inversión en activos, identificar las inversiones en plataformas tecnológicas que se han llevado a cabo en la organización estudiada y finalmente, comparar el retorno de los activos invertidos en tecnología y su incidencia en el desarrollo financiero de la organización de 2016 a 2019.

\section{MARCO TEORICO:}

\section{Naturaleza de la inversión.}

López Rodríguez, et al, (2019) plantean que agregar recursos financieros líquidos con una expectativa de alcanzar unos beneficios, también líquidos, es lo que conoce como inversión y se ejecuta esperando resultados a lo largo de un plazo de tiempo que se denomina vida útil; así mismo es claro que para la ejecución de un proyecto se requiere una inversión, por este motivo es que sin dicha inversión el proyecto quedaría en una simple idea (Reyna, Echeverría \& Borja, 2019). Es por ello, por lo que las diferentes situaciones en una pequeña economía, con riesgo a la volatilidad de los mercados mundiales y con un alto sometimiento a un solo commodity, muestra que la inversión se interpreta como un resultado racional de diferentes agentes económicos (Sachs \& Warner, 2001). 
Rehner \& Rodríguez-Leiva (2017) declara que la rentabilidad positiva que es proyectada por las instituciones financieras es el resultado de ubicar los recursos en actividades de inversión; es por esto que para no arriesgar su capital el inversionista debe efectuar una evaluación que dé como resultado la conveniencia económica del proyecto, de esta manera tendrá la seguridad al realizar la inyección de sus recursos (González, et al, 2017).

Andia (2010) expone que toda empresa u organización, se enfoca en mejorar la calidad de su servicio o producto, es en esta parte en donde necesita dirigir sus proyectos de inversión, por lo cual necesita herramientas financieras para estimar sus posibles resultados. Valencia (2011) plantea que es importante en todas las investigaciones mencionar las opiniones 0 criterios de investigadores, esto entendido en el ámbito empresarial y financiero. Por este motivo una inversión se ejecuta con el fin de lograr productos o beneficios eliminando o comprimiendo varias restricciones al desarrollo de este, generando un incremento de la productividad en un explícito período de tiempo (Bruce, 1982).

González, et al (2017) indican que lo primordial al realizar un proyecto de inversión es identificar el problema, es por esto, por lo que el siguiente paso es cuantificar la necesidad de recursos, por este motivo es transcendente determinar correctamente la demanda insatisfecha del mismo. Por esto mejorar la calidad de servicios o productos es el enfoque de cada empresa, por lo que se hace necesario dirigir los proyectos de inversiones en estas mejoras; la estimación de posibles resultados se da con herramientas financieras (Andia, 2010). De acuerdo con esto la viabilidad de una inversión tiene resultado positivo si crea valor para quién la realiza. Para conocer el valor creado se hace necesario identificar las inversiones que son más costosas en el mercado de lo que cuesta adquirirlas (Martins, Uribe \& Mesa, 2012).
Las teorías de modernización de ideología liberal presumen que un alto nivel de renta exportadora incrementa los ingresos de los hogares, generando una liquidez financiera mayor que se transforma parcialmente en ahorro e inversión privada (Rehner \& Rodríguez-Leiva, 2017). Por este motivo, González et al, (2017) exponen que en cualquier tipo de inversión los rendimientos son factores que van acompañados involuntariamente de las posibilidades técnicas del riesgo. Por este motivo las compañías antes de tomar una decisión financiera deben evaluar las diferentes opciones de créditos que puede encontrar en el mercado y que cubran sus necesidades (López Rodríguez, 2016), así soportar la inversión requerida, logrando generar un mejor soporte a los requerimientos de sus clientes (Campos \& Esquivel, 2019).

Reyna, Echeverría \& Borja (2019) expresan que, al momento de solicitar el financiamiento para la ejecución de una idea, los proyectos de inversión son un elemento fundamental, ya que la idea por sí sola no garantiza el éxito. Es por esto por lo que la financiación para personas de altos ingresos logra simbolizar una innegable "democratización" de la economía, con lo cual es posible participar en rentas derivadas por el capital (Rehner \& Rodríguez-Leiva, 2017). González, et al (2017) argumentan que en una toma de decisiones adecuada contribuyen herramientas 0 instrumentos de evaluación financiera, estos tienen como finalidad determinar los posibles rendimientos minimizando riesgos en la inversión. 


\section{Indicadores financieros.}

Wahyuningsih \& Widowati (2016) exponen que el ROA mide el rendimiento total de los activos después de sacar intereses e impuestos, el rendimiento de los activos nos muestra el buen uso de los mismos para generar ganancias en un periodo de tiempo; así mismo el retorno sobre activos ROA es uno de los índices de rentabilidad más utilizados para medir la capacidad de la gerencia financiera para obtener ganancias, en su conjunto este índice de rentabilidad ilustra la eficiencia del desempeño de la compañía en el retorno de los activos (Iskandar, 2019); dentro de los activos en el análisis del ROA encontramos tangibles e intangibles y que nos permite identificar la inversión del software en la productividad de la empresa (González, D., González, P. \& Roa, 2017).

Si bien el ROA es un indicador que analiza el retorno de la inversión en una empresa, también se puede ver la influencia de sus activos sobre la inversión requerida (Nava \& Martínez, 2012). Dwi (2012) menciona que además de medir la capacidad de la empresa para generar ganancias utilizando sus activos totales que posee la compañía, puede mostrar las variables de crecimiento de inversión dentro de la organización; este indicador de asimetría financiera permite obtener la capacidad de generar riqueza en un activo y es el indicador financiero que elimina el sesgo que existe entre cuentas (Combariza, et al, 2012).

Kurniasari (2017) expone que para calcular la relación del ROA se debe tomar $100 \%$ de ganancias antes de intereses e impuestos sobre los activos totales, cuanto mayor es el ROA, mayor es la media, el nivel de beneficios alcanzado y mejor la posición del resultado. Después de obtener el ROA, se puede medir la tendencia de la empresa y observar el comportamiento de esta en un periodo de tiempo (Combariza, et al 2012). Por ello, Dwi (2012) dice que el capital humano es una determinante ya que este impacta positivamente en los resultados de la compañía por cuan mayor sea su capital intelectual mayor será su rendimiento en los activos.
Este indicador puede definirse como la rentabilidad del activo y mide la rentabilidad de una organización por cada peso invertido en sus activos (Cardona Montoya, et al, 2014); es por esto por lo que los resultados del análisis pueden confirmar la necesidad de la compañía de realizar innovación en sus activos (Martins, Uribe \& Mesa, 2012). Para Uribe, Gaitán \& Potes (2009) la aplicación del análisis de la información financiera no resuelve los problemas de gestión, pero si contribuye a la implementación de nuevas estrategias para el aprovechamiento de los recursos.

Martins, Uribe, \& Mesa (2012) declaran que las empresas con activos con mayor depreciación tienden a tener un ROA relativamente más alto que las empresas con activos de menor depreciación; una empresa trabaja para obtener los mejores resultados utilizando sus activos de manera óptima, se puede observar en el uso de su capital y así lograr ganancias después de impuestos y lo hacen utilizando métodos cuantitativos con los análisis de los estados financieros (Masgrau, 2005); por lo tanto, los inversionistas pueden tener una mayor 0 menor rentabilidad invirtiendo parte de su dinero en activos de alto 0 menor riesgo según los análisis de los mismo (Cardona Montoya, et al, 2014).

La rotación del activo mide las veces que la organización recupera su activo vía ventas, lo que es un indicador eficiente en la utilización de sus activos para generar ingresos (Camarillo, 1997); si bien la rentabilidad del activo se obtiene dividiendo la utilidad neta sobre el activo total, la variación del indicador relaciona la rentabilidad de ventas y la rotación del activo total, lo que permite identificar las áreas responsables del desempeño de rentabilidad del mismo, esto permite evaluar la posición de ganancias de la compañía para un periodo de tiempo (Kurniasari, 2017). 
Wahyuningsih, \& Widowati (2016) expresan que el valor de la compañía se puede medir por medio del precio de las acciones, lo cual es un reflejo del comportamiento en su mercado, entre mayor sea el precio de la acción mayor será la valuación de la empresa. En los análisis de los activos intangibles se pueden encontrar que este tiene gran impacto dentro de la compañía al evaluar el comportamiento frente al ROA (Dwi, 2012).

\section{METODOLOGÍA:}

El enfoque metodológico con el cual se desarrolló el proceso de investigación fue el cualitativo, este permite obtener resultados recolectando y analizando documentación de los fenómenos o partícipes que se están estudiando; lo importante en este enfoque es que el problema de investigación sea medible y su documentación sea clara. Camarillo (1997) indica que en la investigación cualitativa la compresión de la realidad hace parte de los resultados, el investigador capta información real, así como la percibe el sujeto de estudio; por este motivo la orientación cualitativa pretende entender fragmentos de la realidad, tal como se van construyendo en el desarrollo del trabajo y que se irán definiendo por categorías de estudio (De Andrés Pizarro, 2000).

El alcance investigativo fue de tipo descriptivo, desarrollado con la obtención o recolección de documentación financiera real de la compañía a partir de un estudio de caso. Delgado (2001) expresa que los estudios descriptivos radican en narrar o contar las características generales de la distribución, especialmente en relación con personas, lugares y tiempo.
La técnica de estudio de caso facilita el establecer un posible problema constituyendo un potencial análisis de este junto a la descripción de situaciones reales en una compañía, para así llegar a la presentación de posibles soluciones. Es por esto por lo que este método representa una habilidad metodológica de investigación científica, muy útil en la generación de resultados que facilitan el fortalecimiento, evolución y desarrollo de las teorías ya existentes o el aparecimiento de modelos científicos nuevos (Martínez Carazo, 2011).

El estudio de caso se desarrolló mediante la revisión documental y recolección de informes, estados financieros y publicaciones presentadas por la compañía donde se evidencia la inversión tecnológica. Esta información se obtuvo por medio del sitio web institucional de la Superintendencia Financiera de Colombia. Con este proceso se obtuvo la información requerida para el calculo de los indicadores de rentabilidad ROA y ROE, el indicador de endeudamiento y EBITDA, esto llevando a la generación de diferentes alternativas sobre el comportamiento y las causas generadas por la dicha inversión. Barbera \& Inciarte (2012) declaran que la recolección de información sobre actividades, características y resultados permite efectuar reflexiones sobre las actividades realizadas, optimiza la efectividad, o anuncia conclusiones para la toma de decisiones futuras.

\section{RESULTADOS:}

Para el estudio de esta investigación se tuvo en cuenta el estado consolidado de situación financiera expresada en millones de una administradora de fondo de pensiones. Esta información plasmada en la tabla 1 permite ver el estado financiero de la empresa y con ella poder realizar el cálculo de los indicadores estudiados. 
Tabla 1. Estado consolidado de situación financiera (Millones de pesos).

\begin{tabular}{lllll}
\hline \multicolumn{1}{c}{ Activos } & $\mathbf{3 1 / 1 2 / 2 0 1 6}$ & $\mathbf{3 1 / 1 2 / 2 0 1 7}$ & $\mathbf{3 1 / 1 2 / 2 0 1 8}$ & $\mathbf{3 1 / 1 2 / 2 0 1 9}$ \\
\hline Efectivo y equivalentes al efectivo & $\$ 85.068$ & $\$ 39.107$ & $\$ 45.320$ & $\$ 105.055$ \\
\hline Activos financieros de inversión & & & & \\
\hline A valor razonable con cambios en resultados & & & & \\
\hline Inversiones en instrumentos representativos de deuda & $\$ 176.000$ & $\$ 212.287$ & $\$ 168.220$ & $\$ 212.293$ \\
\hline Inversiones en instrumentos de patrimonio & $\$ 1.098 .202$ & $\$ 1.311 .265$ & $\$ 1.375 .184$ & $\$ 1.788 .888$ \\
\hline & $\$ 1.274 .202$ & $\$ 1.523 .552$ & $\$ 1.543 .404$ & $\$ 2.001 .181$ \\
\hline Inversiones a costo amortizado & $\$ 52.362$ & $\$ 78.110$ & $\$ 84.602$ & \\
\hline Activos financieros a valor razonable & & & & \\
\hline Contratos forward de cobertura & $\$-$ & $\$-$ & & $\$-$ \\
\hline Contratos forward de negociación & $\$ 27$ & $\$-$ & $\$ 57$ & $\$-$ \\
\hline
\end{tabular}

A valor razonable con cambios en el ori

\begin{tabular}{lllll}
\hline Inversiones en instrumentos representativos de deuda & $\$-$ & & \\
Cuentas por cobrar & $\$ 45.855$ & $\$ 56.991$ & $\$ 57.577$ & $\$ 47.050$ \\
\hline Activos tangibles, neto & & & & \\
\hline Propiedad y equipo de uso propio y construcciones en curso & $\$ 68.779$ & $\$ 68.042$ & $\$ 85.673$ & $\$ 84.063$ \\
\hline Propiedades de inversión & $\$ 15.330$ & $\$ 17.901$ & $\$ 16.599$ & $\$ 15.355$ \\
\hline Mejoras en propiedades arrendadas & $\$ 2.475$ & & & $\$ 14.959$ \\
\hline \multicolumn{1}{l}{ Activos intangibles } & $\$ 86.584$ & $\$ 85.943$ & $\$ 102.272$ & $\$ 114.377$ \\
\hline Plúsvalia - Crédito mercantil & & & & \\
\hline Otros activos intangibles & $\$ 126.485$ & $\$ 126.485$ & $\$ 126.485$ & $\$ 137.177$ \\
\hline & $\$ 22.659$ & $\$ 13.445$ & $\$ 11.282$ & \\
\hline Activo por impuesto de renta & $\$ 149.144$ & $\$ 139.930$ & $\$ 137.767$ & $\$ 137.177$ \\
\hline Corriente & & & & \\
\hline Diferido & $\$ 264$ & & & \\
\hline & $\$ 128.081$ & $\$ 144.827$ & $\$ 160.879$ & $\$ 178.919$ \\
\hline Otros activos & $\$ 128.345$ & $\$ 144.827$ & $\$ 160.879$ & $\$ 178.919$ \\
\hline Total activos & $\$ 1.153$ & $\$ 2.702$ & $\$ 673$ & $\$ 94$ \\
\hline & $\$ 1.822 .740$ & $\$ 2.071 .162$ & $\$ 2.132 .551$ & $\$ 2.583 .853$ \\
\hline
\end{tabular}


INDICADORES DE RENTABILIDAD, ENDEUDAMIENTO Y EBITDA EN EL ENTORNO DE LA INVERSIÓN EN LAS PLATAFORMAS TECNOLÓGICAS. UN ESTUDIO EN ADMINISTRADORAS DE FONDOS DE PENSIONES

Campo Elías López-Rodríguez - Lina Marcela Pérez-Suarez - Francisco Javier Amado-Quiroga - Yeison Castillo-Areiza

\section{Continua. Tabla 1. Estado consolidado de situación}

financiera (Millones de pesos).

\begin{tabular}{lllll}
\hline Pasivo y patrimonio de los accionistas & $\$ 42.735$ & $\$ 43.100$ & $\$ 43.465$ & $\$ 43.830$ \\
\hline Pasivo & & & & \\
\hline Instrumentos derivativos de cobertura & $\$-$ & & $\$ 409$ & $\$-$ \\
\hline Instrumentos derivativos de negociación & $\$ 14$ & & $\$-$ & $\$-$ \\
\hline Pasivos financieros a valor razonable & $\$ 14$ & $\$-$ & $\$ 409$ & $\$-$ \\
\hline \multicolumn{1}{l}{ Obligaciones laborales } & & & & \\
\hline Beneficios a empleados largo plazo & $\$ 5.162$ & $\$ 6.105$ & & \\
\hline Obligaciones laborales corto plazo & $\$ 23.503$ & $\$ 27.752$ & $\$ 33.713$ & $\$ 39.394$ \\
\hline & $\$ 28.665$ & $\$ 33.857$ & $\$ 33.713$ & $\$ 39.394$ \\
\hline Provisiones & & & & \\
\hline Provisión para contingencias legales & $\$ 12.791$ & $\$ 11.815$ & $\$ 18.388$ & $\$ 22.156$ \\
\hline Seguro previsional & $\$ 341.153$ & $\$ 428.300$ & $\$ 486.289$ & $\$ 569.924$ \\
\hline Otras provisiones & $\$ 2.788$ & $\$ 350$ & $\$ 130$ & $\$ 3.220$ \\
\hline & $\$ 356.732$ & $\$ 440.465$ & $\$ 504.807$ & $\$ 595.300$ \\
\hline Pasivo por impuesto de renta & & & & \\
\hline Corriente & $\$ 57.564$ & $\$ 85.816$ & $\$ 46.169$ & $\$ 60.716$ \\
\hline Diferido & $\$ 34.124$ & $\$ 962$ & & \\
\hline & $\$ 91.688$ & $\$ 86.778$ & $\$ 46.169$ & $\$ 60.716$ \\
\hline Otros pasivos & & & & \\
\hline Cuentas por pagar & $\$ 38.803$ & $\$ 42.131$ & $\$ 44.395$ & $\$ 39.163$ \\
\hline Otros pasivos & $\$ 5.536$ & $\$ 5.706$ & $\$ 5.895$ & $\$ 21.429$ \\
\hline & $\$ 44.339$ & $\$ 47.837$ & $\$ 50.290$ & $\$ 60.592$ \\
\hline Total pasivo & $\$ 521.438$ & $\$ 608.937$ & $\$ 635.388$ & $\$ 756.002$ \\
\hline
\end{tabular}

\begin{tabular}{lllll}
\hline Patrimonio de los accionistas & & & & \\
\hline Capital suscrito y pagado & $\$ 40.347$ & $\$ 40.347$ & $\$ 40.347$ & $\$ 40.347$ \\
\hline Prima en colocación de acciones & $\$ 417.904$ & $\$ 417.904$ & $\$ 417.904$ & $\$ 417.904$ \\
\hline Reservas & $\$ 508.019$ & $\$ 579.707$ & $\$ 739.026$ & $\$ 834.230$ \\
\hline Ganancia o pérdida por diferencia en cambio & $\$ 27.748$ & & & \\
\hline Otros componentes del otro resultado integral & $\$ 18.078$ & $\$ 44.234$ & $\$ 58.316$ & $\$ 57.541$ \\
\hline Utilidad del ejercicio & $\$ 253.170$ & $\$ 343.345$ & $\$ 205.134$ & $\$ 442.790$ \\
\hline Ajustes aplicación primera vez NIIF & $-\$ 12.075$ & & & \\
\hline Ganancias acumuladas de ejercicios anteriores & $\$ 48.111$ & $\$ 36.687$ & $\$ 36.345$ & $\$ 35.038$ \\
\hline Total patrimonio de los accionistas & $\$ 1.301 .302$ & $\$ 1.462 .224$ & $\$ 1.497 .072$ & $\$ 1.827 .850$ \\
\hline Total pasivo y patrimonio de los accionistas & $\$ 1.822 .740$ & $\$ 2.071 .161$ & $\$ 2.132 .460$ & $\$ 2.583 .852$
\end{tabular}

Fuente: Elaboración propia con base en información de la Superintendencia Financiera (2019). 
De igual manera fue necesario obtener el estado consolidado de resultados expresado en millones de la misma entidad, vistos en la tabla 2. El estado de resultados muestra las ganancias o pérdidas, el detalle de como obtuvo el resultado de la operación de la empresa, por tanto, con estos datos suministrados se pudo calcular los indicadores estudiados.

Tabla 2. Estado consolidado de resultados (Millones de pesos).

\begin{tabular}{|c|c|c|c|c|}
\hline Ingresos, neto por operaciones ordinarias & $31 / 12 / 2016$ & $31 / 12 / 2017$ & $31 / 12 / 2018$ & $31 / 12 / 2019$ \\
\hline Ingresos por comisiones y honorarios & $\$ 802.751$ & $\$ 868.522$ & $\$ 880.739$ & $\$ 924.836$ \\
\hline Gastos por comisiones y honorarios & $\$ 55.258$ & $\$ 60.687$ & $\$ 59.110$ & $\$ 61.620$ \\
\hline $\begin{array}{l}\text { Total ingresos, neto por comisiones y } \\
\text { honorarios }\end{array}$ & $\$ 747.493$ & $\$ 807.835$ & $\$ 821.629$ & $\$ 863.216$ \\
\hline \multicolumn{5}{|l|}{ Otros ingresos } \\
\hline $\begin{array}{l}\text { Ganancia neta sobre instrumentos financieros de } \\
\text { patrimonio }\end{array}$ & $\$ 89.635$ & $\$ 162.578$ & $-\$ 2.440$ & $\$ 241.346$ \\
\hline Ganancia neta por valoración a costo amortizado & $\$ 222$ & $\$ 8$ & $\$ 10$ & $\$ 3$ \\
\hline Ganancia neta por diferencia en cambio & $\$ 234$ & $-\$ 5.191$ & $\$ 215$ & $\$ 545$ \\
\hline Otros ingresos & $\$ 18.969$ & $\$ 27.854$ & $\$ 8.725$ & $\$ 7.771$ \\
\hline Total otros ingresos neto & $\$ 109.060$ & $\$ 185.249$ & $\$ 6.510$ & $\$ 249.665$ \\
\hline \multicolumn{5}{|l|}{ Ingreso por intereses } \\
\hline $\begin{array}{l}\text { Ganancia neta sobre instrumentos financieros de } \\
\text { deuda }\end{array}$ & $\$ 14.760$ & & & $-\$ 1.079$ \\
\hline Otros intereses & $\$ 4.127$ & $\$ 2.437$ & $\$ 3.266$ & $\$ 2.453$ \\
\hline Total ingresos por intereses & $\$ 18.887$ & $\$ 2.437$ & $\$ 3.266$ & $\$ 1.374$ \\
\hline \multicolumn{5}{|l|}{ Gastos por intereses y similares } \\
\hline Obligaciones financieras & $\$ 669$ & $\$ 886$ & $\$ 510$ & $\$ 2.375$ \\
\hline Otros intereses & $\$ 1.314$ & $\$ 80$ & & \\
\hline Total gastos por intereses y similares & $\$ 1.983$ & $\$ 966$ & $\$ 510$ & $\$ 2.375$ \\
\hline \multicolumn{5}{|l|}{ Pérdidas por deterioro de activos financieros } \\
\hline Provisiones & $\$ 81.784$ & $\$ 99.176$ & $\$ 65.666$ & $\$ 101.469$ \\
\hline \multicolumn{5}{|l|}{ Gastos de administración } \\
\hline Gastos de personal & $\$ 152.254$ & $\$ 170.946$ & $\$ 169.227$ & $\$ 177.124$ \\
\hline Gastos Honorarios & $\$ 45.913$ & $\$ 51.571$ & $\$ 56.826$ & $\$ 67.937$ \\
\hline Impuestos & $\$ 10.099$ & $\$ 10.612$ & $\$ 10.883$ & $\$ 10.636$ \\
\hline Gastos generales de administración & $\$ 41.006$ & $\$ 41.778$ & $\$ 57.291$ & $\$ 48.316$ \\
\hline Gastos por depreciación y amortización & $\$ 10.522$ & $\$ 15.701$ & $\$ 13.481$ & $\$ 12.442$ \\
\hline Otros egresos & $\$ 105.549$ & $\$ 108.607$ & $\$ 115.011$ & $\$ 119.188$ \\
\hline Total gastos de administración & $\$ 365.343$ & $\$ 399.215$ & $\$ 422.719$ & $\$ 435.643$ \\
\hline $\begin{array}{l}\text { Utilidad antes de impuestos sobre la renta y } \\
\text { CREE }\end{array}$ & $\$ 426.330$ & $\$ 496.164$ & $\$ 342.510$ & $\$ 574.768$ \\
\hline Gasto de impuesto de renta y CREE & $\$ 173.160$ & $\$ 152.819$ & $\$ 137.376$ & $\$ 131.978$ \\
\hline Utilidad neta del ejercicio & $\$ 253.170$ & $\$ 343.345$ & $\$ 205.134$ & $\$ 442.790$ \\
\hline
\end{tabular}

Fuente: Elaboración propia, con base en información de la Superintendencia Financiera (2019). 
Al identificar la información financiera anteriormente expuesta y analizar los indicadores de rentabilidad ROA y ROE, indicadores de endeudamiento y EBITDA, generados por la inversión de plataformas tecnológicas en una organización del sector financiero colombiano por medio de un estudio de caso, se evidencia que la compañía efectúo inversiones en relanzamiento de la página web, actualización de servicios en la plataforma web y por último invirtió en servicios tecnológicos como los puntos de atención rápida para sus afiliados.

Todas estas inyecciones de capital dieron como resultado que, el ROA divide la utilidad neta por los activos, midiendo el crecimiento de la empresa, el cual para el año 2016 fue del 14\%, en 2017 fue del 17\%, en 2018 el resultado fue $10 \%$ y en $2019,17 \%$. Este incremento del $7 \%$ se ve sustentado en el último año dado que la compañía decidió invertir durante este periodo como se muestra en la tabla 3.

Tabla 4. ROE.

\begin{tabular}{ccccc}
\hline ROE & $31-12-16$ & $31-12-17$ & $31-12-18$ & $31-12-19$ \\
\hline $\begin{array}{c}\text { Utilidad } \\
\text { neta/patrimonio }\end{array}$ & $19 \%$ & $23 \%$ & $14 \%$ & $24 \%$ \\
\hline
\end{tabular}

Fuente: Elaboración propia.

El nivel de endeudamiento en el año 2016 fue 28.61\%, en el 2017 el porcentaje incrementó llegando al $29.40 \%$, en 2018 aumento nuevamente resultando $29.79 \%$ y al periodo financiero de 2019 disminuyo al $29.26 \%$, este indicador mide que tanto es utilizado el apalancamiento financiero para aumentar la rentabilidad esperada. Lo anterior sustentado en la tabla 5.

Tabla 5. Nivel de endeudamiento.

\begin{tabular}{ccccc}
\hline ENDEUDAMIE & $\begin{array}{c}31-12- \\
\text { NTO }\end{array}$ & $\begin{array}{c}31-12- \\
\mathbf{3}\end{array}$ & $\begin{array}{c}31-12- \\
18\end{array}$ & $\begin{array}{c}31-12- \\
19\end{array}$ \\
\hline Pasivo & 28,61 & 29,40 & 29,79 & 29,26 \\
total/activo & $\%$ & $\%$ & $\%$ & $\%$ \\
total & & & & \\
\hline
\end{tabular}

Fuente: Elaboración propia.
Concluyendo, el ultimo indicador analizado e informado conceptualmente es el EBITDA, el cual es generado de tomar los ingresos, restándole costos de ventas, deduciéndole gastos de administración para así sumarle la depreciación y amortización; en el año 2016 da un valor de $\$ 593^{\prime} 069.000$ millones de pesos, en 2017 , incremento un $9 \%$ llegando a $\$ 647^{\prime} 782.000$ millones de pesos, para el año 2018 disminuyo un -4\% siendo $\$ 619^{\prime} 601.000$ millones de pesos y en 2019 aumenta $5 \%$ dando un valor de $\$ 651^{\prime} 672.000$ millones de pesos. Lo anterior sustentado en la tabla 6.

Tabla 6. EBITDA.

\begin{tabular}{|c|c|c|c|c|}
\hline EBITDA & $\begin{array}{l}31- \\
12- \\
16\end{array}$ & $\begin{array}{l}31- \\
12- \\
17\end{array}$ & $\begin{array}{l}31- \\
12- \\
18\end{array}$ & $\begin{array}{l}31- \\
12- \\
19\end{array}$ \\
\hline Ingresos & $\begin{array}{r}821 . \\
720\end{array}$ & $\begin{array}{r}896 . \\
376\end{array}$ & $\begin{array}{r}889 . \\
464\end{array}$ & $\begin{array}{r}932 . \\
607\end{array}$ \\
\hline - Costo de ventas & - & - & - & - \\
\hline $\begin{array}{l}\text { - Gastos de } \\
\text { administración y } \\
\text { ventas }\end{array}$ & $\begin{array}{r}239 . \\
173\end{array}$ & $\begin{array}{r}264 . \\
295\end{array}$ & $\begin{array}{r}283 . \\
344\end{array}$ & $\begin{array}{r}293 . \\
377\end{array}$ \\
\hline $\begin{array}{l}\text { + Depreciación + } \\
\text { amortización }\end{array}$ & $\begin{array}{r}10.5 \\
22\end{array}$ & $\begin{array}{r}15.7 \\
01\end{array}$ & $\begin{array}{r}13.4 \\
81\end{array}$ & $\begin{array}{r}12.4 \\
42\end{array}$ \\
\hline \multirow[t]{2}{*}{ = Ebitda } & $\begin{array}{r}593 . \\
069\end{array}$ & $\begin{array}{r}647 . \\
782\end{array}$ & $\begin{array}{r}619 . \\
601\end{array}$ & $\begin{array}{r}651 . \\
672\end{array}$ \\
\hline & & $9 \%$ & $-4 \%$ & $5 \%$ \\
\hline
\end{tabular}

Fuente: Elaboración propia.

\section{DISCUSIÓN Y CONCLUSIONES:}

El desarrollo de la investigación permitió analizar y relacionar los conceptos de los indicadores de rentabilidad, endeudamiento y EBITDA, identificando las inversiones en plataformas tecnológicas, logrando generar una comparación del retorno de los activos invertidos en tecnología junto a su incidencia en el desarrollo financiero de la organización entre un periodo de tiempo a través de diversos indicadores financieros, siendo este el objetivo de toda gerencia financiera al momento de generar valor, lo que permite 
brindar crecimiento en las organizaciones y para eso se deben establecer resultados con frecuencia utilizando diferentes indicadores de financieros (Wahyuningsih, \& Widowati, 2016; García, 2018; Orozco \& Rodríguez, 2017).

De igual manera, la investigación dio a conocer resultados financieros sobre la inversión realizada por la empresa en sus plataformas tecnológicas, de ahí que para conocer el resultado de una inversión se hace necesario tener en cuenta un período de tiempo inferior a la vida útil real del proyecto (Reyna, Echeverría \& Borja, 2019; Rodríguez, Luengas \& Díaz, 2017). Así mismo, se tuvo en cuenta un espacio de tiempo, siendo este un ciclo de los últimos 4 años, específicamente del año 2016 al 2019. De esta manera, se comparó el retorno de los activos invertidos y se analizaron financieramente los ingresos, costos y gastos generados durante ese periodo de tiempo.

Uno de los indicadores analizados en esta investigación fue el ROA, el cual muestra la eficiencia de activos que se maneja en la compañía ayudando a confrontar el beneficio obtenido por la misma, contra el total de activos de la organización en sus últimos periodos contables (Andrade-Pinelo, 2011; Kurniasari, 2017; Combariza, et al, 2012). Al analizar los estados financieros de un caso real, se logró comparar el retorno de activos durante los últimos 4 años, evidenciando un crecimiento porcentual en el último periodo del ROA, donde se demuestra que la organización obtuvo mayor rentabilidad en el último ciclo sobre los otros tiempos en el total de sus activos.

Otro de los indicadores analizados fue el ROE, el cual surge del margen de utilidad, del nivel de manejo de activos $\mathrm{y}$, por último, del apalancamiento financiero que se aplique en el año contable (Nava \& Martínez 2012). Referente a esto, se evidencia que la rentabilidad de la compañía sobre fondos propios tuvo un crecimiento comparando los 2 últimos años, concluyendo que los activos fueron mayores gracias a su bajo nivel de endeudamiento y/o apalancamiento, el cual fue estable en los periodos evaluados.
Dentro de las limitaciones que se pueden encontrar las organizaciones al momento de buscar crecimiento financiero se evidencia un inadecuado manejo en el porcentaje de apalancamiento financiero o deuda que presentan; de ahí la pertinencia de los indicadores relacionados con estas actividades, puesto que su principal función dentro del proceso administrativo es la de medir y tomar decisiones de acuerdo con sus resultados. El endeudamiento se expresa de manera positiva al momento en el que el nivel de apalancamiento aumenta en la empresa su rentabilidad financiera. En este caso, se muestra cómo el porcentaje de apalancamiento es bajo, considerándose positivo siendo estable en los últimos cuatro años, sustentando la rentabilidad sobre los activos y la rentabilidad sobre el patrimonio, la cual creció como se evidenció en los resultados financieros.

Un tema que no se debe dejar atrás en la evaluación financiera que deben tener en cuenta las organizaciones es el análisis del EBITDA, que muestra los resultados financieros, pero por sí solo no es suficiente medida para indicar si un proyecto es rentable o no, por lo cual es necesario evaluar junto con otros indicadores que valoren los demás aspectos financieros sensibles del proyecto. El EBITDA evidenciado en este caso, determina que la organización generó mayores ingresos y menos gastos en los últimos años, y por ende el proyecto es rentable.

El desarrollo metodológico fue idóneo para cumplir con los objetivos planteados en el desarrollo de la investigación, esto se evidencia desde la identificación de los indicadores propuestos, visualizando las inversiones de la organización y sus ganancias reales. Los resultados obtenidos aportan conocimiento financiero sobre una de las administradoras de fondo de pensiones y cesantías más grande del país exponiendo las maneras en las cuales crecen día tras día en términos financieros. 


\section{REFERENCIAS:}

Andia, W. (2010). Proyectos de inversión: un enfoque diferente de análisis. (En línea). PE. Industrial Data UNMSM, 13(1), 4-13.

Andrade-Pinelo, A. M. (2011). Análisis del ROA, ROE y ROI. Contadores y Empresas, 170, 59-61.

Barbera, N., \& Inciarte, A. (2012). Fenomenología y hermenéutica: dos perspectivas para estudiar las ciencias sociales $y$ humanas. Multiciencias, 12(2), 199-205.

Bruce, C. F. (1982). The Project Cycle. An introduction to the stage of project planning and implementation. Banco Mundial, Instituto de Desarrollo Económico, CN347a, 1-5.

Campos, C. L. \& Esquivel, G. M. (2019). Análisis de las tasas de interés de referencia en Costa Rica, en moneda nacional y extranjera, desde una perspectiva de inversión financiera utilizando la metodología del Efecto Fischer Internacional. Economía y Sociedad, 24(56), 1-34.

Cardona Montoya, R., Velasquez, H., Vidal, T., \& Escobar, R. (2014). APT-Evidencia Empírica En El Análisis Del ROA En Una Empresa De Servicios Públicos Domiciliarios De Acueducto $Y$ Alcantarillado. Center for Research in Economics and Finance (CIEF), Working Papers, (14-25).

Camarillo, G. (1997). Confiabilidad y validez en estudios cualitativos. Educación y ciencia, 1(15), 77-82.
Combariza, N., García, C., Alvarado, L., España, C., \& Rivera, H. (2012). Análisis estratégico del sector de Telecomunicaciones: empaquetamiento tecnológico. Documentos de Investigación Facultad de Administración, 132(132), 1-36.

De Andrés Pizarro, J. (2000). El análisis de estudios cualitativos. Aten Primaria, 25(1), 42-46.

Delgado, D. C. (2001). Estrategias de investigación. Diseños observacionales 1 parte. Estudios descriptivos. La Revista Chilena, 53(2), 229233.

Dwi, D. A. (2012). Pengaruh intellectual capital terhadap Return on Asset (ROA) perbankan. Nominal, Barometer Riset Akuntansi dan Manajemen, 1(1), 34-40.

García, J. P. (2018). ¿Puede utilizarse el EBITDA de forma oportunista?: Desviaciones detectadas en la aplicación del EBITDA normalizado de AECA. AECA: Revista de la Asociación Española de Contabilidad y Administración de Empresas, (122), 3-6.

González, C. O., Valarezo, J. K., Montero, V. A. \& Sarmiento, C. B. (2017). Perspectivas de los criterios de evaluación financiera, una selfie al presupuesto de proyectos de inversión. INNOVA Research Journal, 2(8.1), 139-158.

González, D. P., González, P. S. \& Roa, K. D. (2017). Análisis del impacto de las TIC en la productividad de las empresas del IBEX 35 , España y Col20, Colombia. Puente, 7(1), 1727. 
Iskandar, I. (2019). Analisis vector autoregression (var) terhadap Interrelationship antara financing deposit ratio (fdr) dan Return on asset (roa) pada bank syariah di indonesia. Jurnal Ekonomi Syariah, Akuntansi dan Perbankan (JESKaPe), 3(2), 19-39.

Kurniasari, R. (2017). Analisis Return on Assets (ROA) dan Return On Equityl Terhadap Rasio Permodalan (Capital Adequacy Ratio) Pada PT Bank Sinarmas Tbk. MoneterJurnalAkuntansi dan Keuangan, 4(2), 150158..

López Rodríguez, C. E. (2016). El gerente competitivo y su rol en las mipymes: una perspectiva desde el marketing. Equidad y Desarrollo, (25).

López Rodríguez, C. E., Cadena López, S., Montenegro Castillo, D. C., \& Plata Niño, J. A. (2019). Incidencia de la banca multilateral en las asociaciones público-privadas: una perspectiva en Colombia. Equidad y Desarrollo, 1(33), 235-253.

López-Rodríguez, C. E., Cardozo, C. E., Monroy, L. \& González, E. G. (2019). El impacto del valor económico agregado (EVA) en la valoración accionaria de las empresas colombianas entre los años 2015 al 2018. Revista ESPACIOS, 40(43).

Lopez-Rodriguez, C. E., Cristancho-Triana, G. J., \& Amaya-Tellez, J. L. (2020). Perception and Desired Brand Personality in Organizations that Internationalize Services: The Case of the Colombian Business Environment. European Research Studies Journal, 23(3), 581-597.
Martins, I., Uribe, F., \& Mesa, D. (2012). Contribución de la orientación emprendedora a la rentabilidad de las pymes: un análisis contingente considerando la función del entorno. Ecos de Economía: A Latin American Journal of Applied Economics, 16(35), 45-71.

Martínez Carazo, P. C. (2011). El método de estudio de caso Estrategia metodológica de la investigación científica. Revista científica Pensamiento y Gestión, (20).

Masgrau, E. G. (2005). El apalancamiento financiero: de cómo un aumento del endeudamiento puede mejorar la rentabilidad financiera de una empresa. Revista de contabilidad y dirección, 2, 71-91.

Nava, A. R., \& Martínez, F. V. (2012). Liquidez y apalancamiento de la banca comercial en México. Análisis Económico, 27(66), 73-96.

Orozco, Á. A. \& Rodríguez, C. E. (2017). El Copazú y los negocios inclusivos, una estrategia socioeconómica en FlorenciaCaqueta. Cooperativismo Desarrollo, 25(112), 3-28.

Pérez-Rave, J., Ocampo, F. \& Restrepo, G. (2018). RentabiLean: Modelo de exploración del efecto de iniciativas Lean sobre la rentabilidad financiera. Ingeniería, investigación y tecnología, 19(1), 89-99.

Rehner, J. \& Rodríguez-Leiva, S. (2017). Inversión inmobiliaria en tiempos de auge y crisis: ¿Es la ciudad un producto minero o un derivado financiero? Revista de Geografía Norte Grande, (67), 183-210. 
Reyna, F. R., Echeverría, G. C. \& Borja, G. F. (2019). La evaluación de proyectos de inversión: una perspectiva financiera. Opuntia Brava, 11(Especial 2), 465-473.

Rodríguez, C. E., Luengas, B. A. \& Díaz, E. L. (2017). Tendencias de consumo y de la distribución en el mercado de las arepas de maíz en la ciudad de Bogotá DC. Revista CIFE: Lecturas de Economía Social, 19(30), 149-172.

Sachs, J. D., \& Warner, A. M. (2001). The curse of natural resources. European economic review, 45(4-6), 827-838.

Superintendencia Financiera (2018). Afiliados Superintendencia financiera de Colombia. Recuperado de https://www.superfinanciera.gov.co/jsp/9110

Uribe, R. P., Gaitán, M. G. \& Potes, M. N. (2009). Análisis Empírico de la aplicación del modelo de modernización de la gestión para organizaciones en pymes colombianas. Revista Escuela de administración de negocios, (65), 77-105.

Valencia, W. A. (2011). Indicador de rentabilidad de proyectos: el valor actual neto (van) o el valor económico agregado (eva). Industrial data, 14(1), 15-18.

Wahyuningsih, P. \& Widowati, M. (2016). Analisis ROA dan ROE Terhadap Nilai Perusahaan dengan Corporate Social Responsibility Sebagai Variabel Moderating (StudiPada Perusahaan BUMN yang Terdaftar Di BEI Periode 20102013). Jurnal STIESemarang, 8(3), 83-102. 